\title{
Muscular involvement and tendon contracture in limb-girdle muscular dystrophy 2 Y: a mild adult phenotype and literature review
}

\author{
Xuelin Feng ${ }^{1}$, Jinlang $\mathrm{Wu}^{2}$, Wenbiao Xian ${ }^{1}$, Bing Liao ${ }^{3}$, Songjie Liao ${ }^{1}$, Xiaoli Yao ${ }^{1}$ and Weixi Zhang ${ }^{1 *}$ (D
}

\begin{abstract}
Background: Limb girdle muscular dystrophy type $2 Y$ (LGMD2Y) is a rare subgroup of limb girdle muscular dystrophy featuring limb-girdle weakness, tendon contracture and cardiac involvement. It is caused by the mutation of TOR1AIP1, which encodes nuclear membrane protein LAP1 (lamina-associated polypeptide 1) and comprises heterogeneous phenotypes. The present study reported a patient with a novel homozygous TOR1AIP1 mutation that presented with selective muscle weakness, which further expanded the phenotype of LGMD2Y- and TOR1AIP1-associated nuclear envelopathies.

Case presentation: A 40-year-old male presented with Achilles tendon contracture and muscle weakness that bothered him from 8 years old. While the strength of his distal and proximal upper limbs was severely impaired, the function of his lower limbs was relatively spared. Muscle pathology showed dystrophic features, and electron microscopy showed ultrastructural abnormalities of disrupted muscle nuclei envelopes. Whole-exome sequencing showed a frameshift mutation in TOR1AIP1 (c.98dupC).

Conclusion: We reported a novel mild phenotype of LGMD2Y with relatively selective distal upper limb weakness and joint contracture and revealed the heterogeneity of LGDM2Y and the role of the LAP1 isoform by literature review.
\end{abstract}

Keywords: LGM2Y, LAP1, Nuclear envelopathies, Tendon contracture, Case report

\section{Background}

Limb-girdle muscular dystrophy (LGMD) is one of the most common muscular diseases featuring proximal muscle weakness [1]. However, some LGMD patients also present distal muscle weakness and tendon contracture, making it difficult to establish a diagnosis. LGMD2Y is a special subgroup of LGMD that presents

\footnotetext{
* Correspondence: zhangwxi@mail.sysu.edu.cn

'Department of Neurology, The First Affiliated Hospital, Sun Yat-sen University; Guangdong Provincial Key Laboratory of Diagnosis and Treatment of Major Neurological Diseases, National Key Clinical Department and Key Discipline of Neurology, No.58 Zhongshan Road 2, Guangzhou 510080, China Full list of author information is available at the end of the article
}

distal weakness, joint contracture, restrictive lung disease and cardiac involvement in addition to limb-girdle muscle weakness [2]. LGMD2Y has been reported in Turkey [2], Australia [3], the USA [4] and Germany [5]. It is an autosomal recessive-inherited disease caused by a mutation in the torsinA-interacting protein 1 (TOR1AIP1) gene [2], which encodes lamina-associated polypeptide 1 (LAP1), a nuclear envelope protein [6]. It is a lamina-binding protein and is related to maintaining the nuclear membrane, and its defect is related to muscular dystrophy, dystonia and multisystem disorder [7].

(c) The Author(s). 2020 Open Access This article is licensed under a Creative Commons Attribution 4.0 International License, which permits use, sharing, adaptation, distribution and reproduction in any medium or format, as long as you give appropriate credit to the original author(s) and the source, provide a link to the Creative Commons licence, and indicate if changes were made. The images or other third party material in this article are included in the article's Creative Commons licence, unless indicated otherwise in a credit line to the material. If material is not included in the article's Creative Commons licence and your intended use is not permitted by statutory regulation or exceeds the permitted use, you will need to obtain permission directly from the copyright holder. To view a copy of this licence, visit http://creativecommons.org/licenses/by/4.0/ The Creative Commons Public Domain Dedication waiver (http://creativecommons.org/publicdomain/zero/1.0/) applies to the data made available in this article, unless otherwise stated in a credit line to the data. 
The present study reported an adult Chinese pedigree with a novel homozygous TOR1AIP1 mutation, who presents joint contracture, distal weakness and proximal weakness with upper limb predominance. This pure muscular and tendon-involved presentation further expands the phenotype of TOR1AIP1-associated nuclear envelopathies.

\section{Case presentation}

\section{Patient recruitment and consent}

The study included a patient who was admitted to the Department of Neurology, the First Affiliated Hospital, Sun Yat-sen University, in February 2019. Written informed consent for participation was obtained from the patient. The study was approved under the guidelines of the Institutional Ethics Committee of the First Affiliated Hospital of Sun Yat-Sen University.

\section{Clinical features of LGMD2Y}

The patient was a 40-year-old male from a consanguineous family in Northwest China who suffered from muscle weakness and tendon contracture (Fig. 1a). The patient complained of muscle weakness in the proximal lower limbs and upper limbs at 8 years old. He complained of disability in jumping or running fast in childhood. His distal upper limbs were preferentially impaired. He was unable to extend the wrist and fingers (Current Medical Research Council (MRC) score: 1, Fig. 1b). Gradually, the proximal upper limbs had progressive muscle weakness with preserved muscle strength in the lower limbs. At age 20 years, he was unable to raise the arms. Currently, the Gardner-Medwin and Walton modified scale of clinical severity score (GMW) [8] score is 1, and his 6-min walk test [9] result is $565 \mathrm{~m}$. However, the strength of the upper limbs decreased severely with dominant proximal muscle atrophy (Fig. 1c). His Brooke Upper Extremity Rating Scale [10] was grade 5. The performance of the upper limb (PUL) [11] score of his shoulder, elbow and hand/finger was 6, 16 and 11, respectively. The strength of neck flexion was mildly decreased (MRC score: 4), while neck extension was spared.

Regarding tendon contracture, Achilles tendon contracture was revealed, while interphalangeal joints, elbow and spine were spared. Laboratory examination showed that creatine kinase levels were mildly elevated $(230 \mathrm{U} / \mathrm{L})$ and lactate dehydrogenase levels were normal. In the never conduction test, the conduction velocity and $\mathrm{F}$ wave were normal in each limb. In the electromyography test, no spontaneous potential was found, and several polyphasic motor unit potentials with short durations were documented.

\section{Pathological finding}

The muscle biopsy showed dystrophic features (Fig. 2a). Irregular and hypertrophic fibres were found. The fibrosis of the perimysium and endomysium was increased. Split fibres were observed. Some muscle fibres had increased internal nuclei. Degenerating and necrotic fibres with mild local inflammatory infiltration were observed. Disorganized muscle fibre structure was identified by nicotine adenine dinucleotide tetrazolium reductase (NADH-TR) staining (Fig. 2b). In addition, reduced cytochrome $\mathrm{C}$ oxidase activity was also found in some fibres (Fig. 2c). In modified Gomori trichrome staining, aggregation was found in a few muscle fibres (Fig. 2d). Oil Red O, periodic acid-Schiff, ATPase, dystrophin,
A

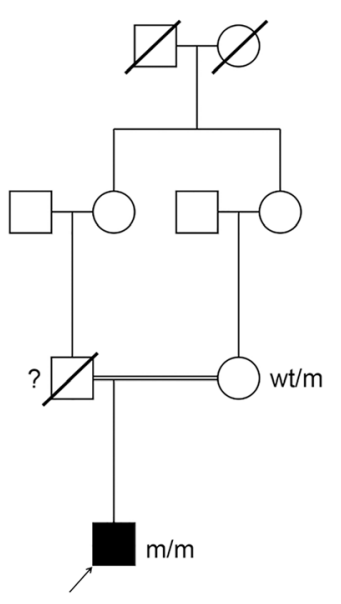

B

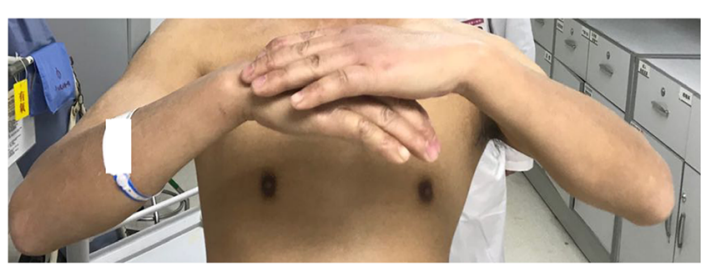

C

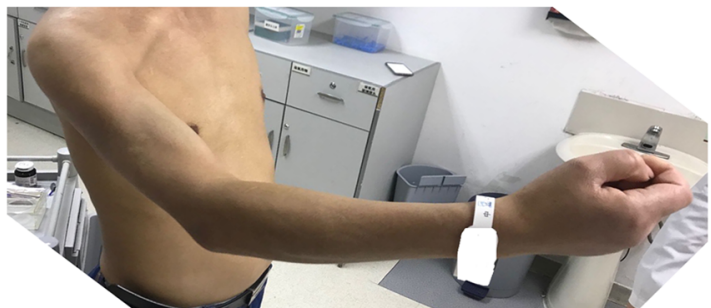

Fig. 1 Family pedigree and clinical presentation of the present patient. (A) Family pedigree. DNA samples were obtained from the proband and his mother. They were tested by next-generation sequencing and Sanger sequencing, respectively. (B-C) Sign of upper limb weakness of the present patient. He could not extend his wrist and fingers (B). His proximal muscle of the upper limbs was atrophied (C) 


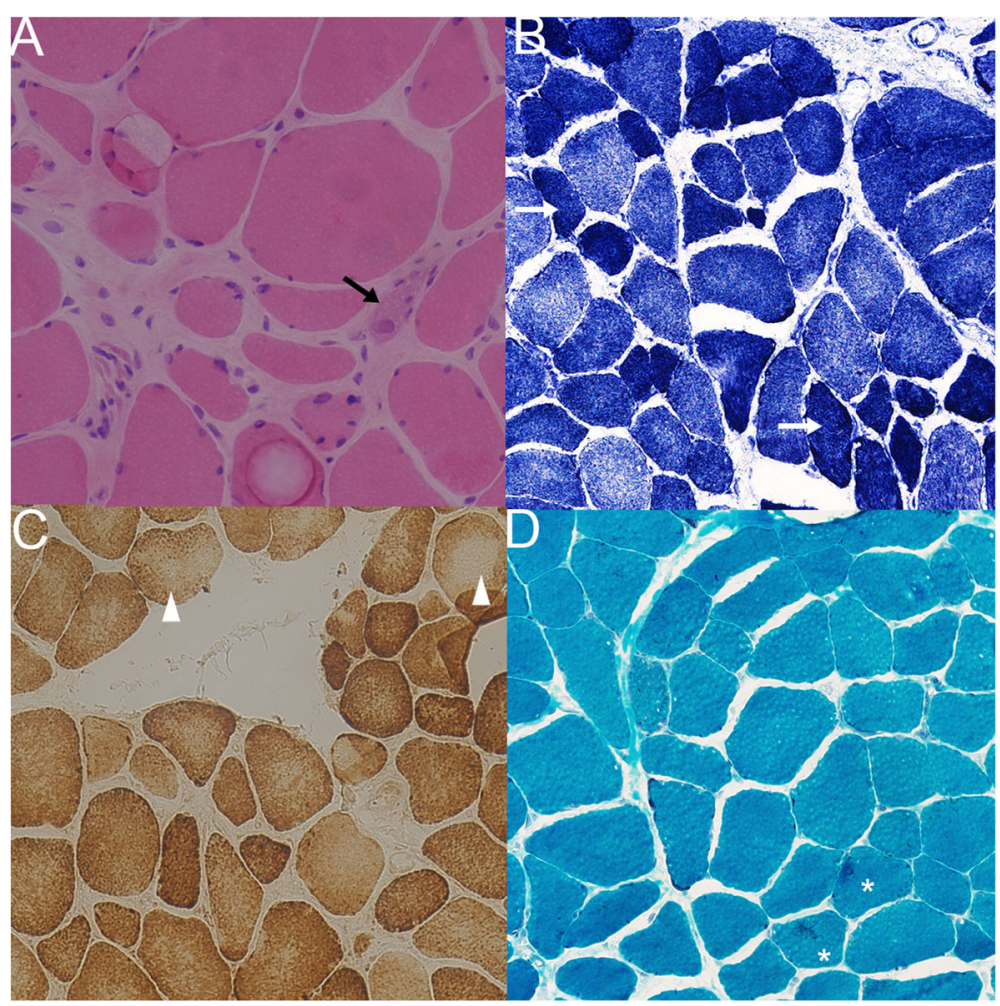

Fig. 2 Histopathological findings of the present patient (magnification: $20 \times 10$ ). (A) Haematoxylin and eosin staining of the present patient. Muscle fibres were irregular and varied in size. Necrotic fibre (black arrow) with mild inflammatory infiltration was found. (B) Nicotine adenine dinucleotide tetrazolium reductase staining of the present patient. The internal architecture of some muscle fibres was damaged (white arrow). (C) Cytochrome C oxidase staining of the present patient. Reduced cytochrome C oxidase activity was found in some fibres (arrowhead). (D) Modified Gomori's trichrome staining of the present patient. Aggregation in muscle fibre was found (*)

dysferlin and major histocompatibility complex 1 staining were normal.

In transmission electron microscopy, ultrastructural abnormalities of muscle nuclei were found, including multiple nuclei, chromatin condensation, segmented nuclei and naked chromatin with a disrupted envelope (Fig. 3a-c). Increased lysosomes were identified in the subsarcolemmal area (Fig. 3d). The structure of the myofibril was spared, and no deposition was found.

\section{Genetic analysis}

Whole-exome sequencing was used to establish a genetic diagnosis. It was performed on a NextSeq 500 (Illumina, Inc., USA). Burrows-Wheeler Aligner (version 0.7.10) was used for read mapping [12], and the Genome Analysis Toolkit (version 4.0.8.1) was used for adjustment and discovery of SNPs, insertions and deletions [13]. Annotation was carried out by Annovar (version: 2018-0416) [14]. A novel homozygous mutation in TOR1AIP1 was found (c.98dupC, Fig. 4), leading to a frameshift mutation (p.Q35Sfs*74). Its frequency was examined in population databases, including the 1000 Genomes Project (https://www.internationalgenome.org/), the NHLBI
Exome Sequencing Project (https://evs.gs.washington. edu/EVS/) and The Exome Aggregation Consortium (http://exac.hms.harvard.edu/). This mutant was classified as "likely pathogenic" according to the American College of Medical Genetics and Genomics standards (PSV1 + PM2) [15]. Sanger sequencing of the patient's mother revealed the same mutation in TOR1AIP1 (a sample from the patient's father was not available).

\section{Discussion and conclusion}

TOR1AIP1-associated nuclear envelopathies comprise a spectrum of phenotypes ranging from a severe infantile form with multiple system involvement to a mild form of pure tendon and muscle involvement such as LGMD2Y. Our study presented an adult case with mild limb girdle involvement as well as distal muscle weakness due to a novel N-terminal frameshift mutation. This relatively mild adult phenotype further expands the understanding of genotype-phenotype correlation and provides diagnostic clues in neurological assessment for LGMD.

LGMD2Y is a rare subgroup of autosomal recessive LGMD, and only a few cases have been reported (Table 1) [2-5]. We reviewed the literature and summarized the 


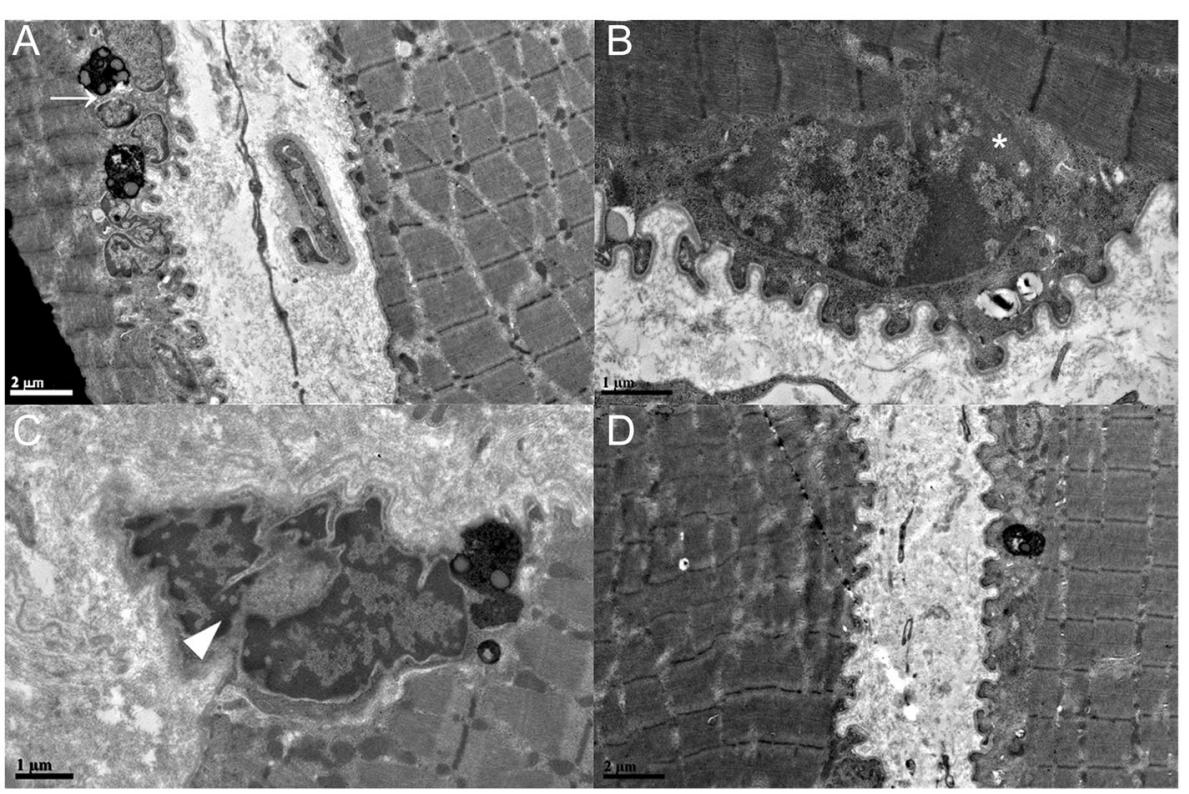

Fig. 3 Ultrastructural findings of the present patients. (A) Multiple nuclei (arrow), chromatin condensation and segmented nuclei. (B) Nuclear membrane disruption and naked chromatin (*). (C) Chromatin condensation and broken nucleus (arrowhead). (D) Normal structure of sarcomeres

phenotypes and genotypes. Most cases had disease onset in the first or second decade and insidiously progressed. The main clinical presentations include proximal weakness and atrophy of limbs and tendon contracture. The most common tendon contracture was contracture of the Achilles tendon (4/6) and interphalangeal joints (4/6). Other signs include rigid spine and kyphoscoliosis. Some of the patients presented with distal weakness and atrophy of limbs (4/8). Cardiac involvement (5/7) and restrictive lung disease $(4 / 5)$ were common. Notably, four cases of dilated myocardiopathy have been reported, and most of them require heart transplantation or die due to heart failure $[3,5]$. Only one muscle magnetic resonance imaging showed selective fatty infiltration in the medial and posterior parts of the thigh [4]. In contrast to a previous report, our case of LGDM2Y showed dominant impairment of the upper limbs, especially the muscle of the distal upper limbs. While he was still to retain the function of

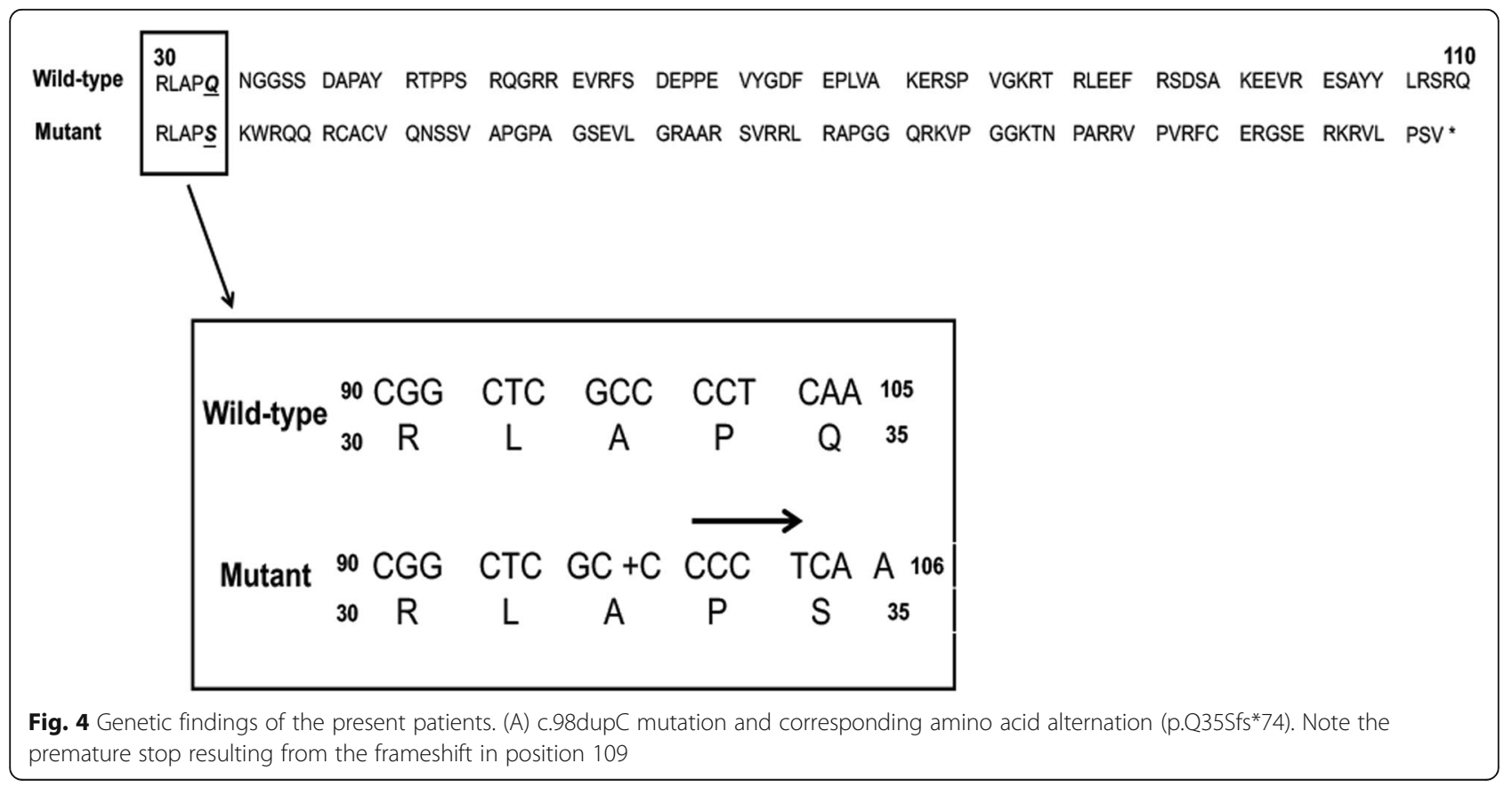




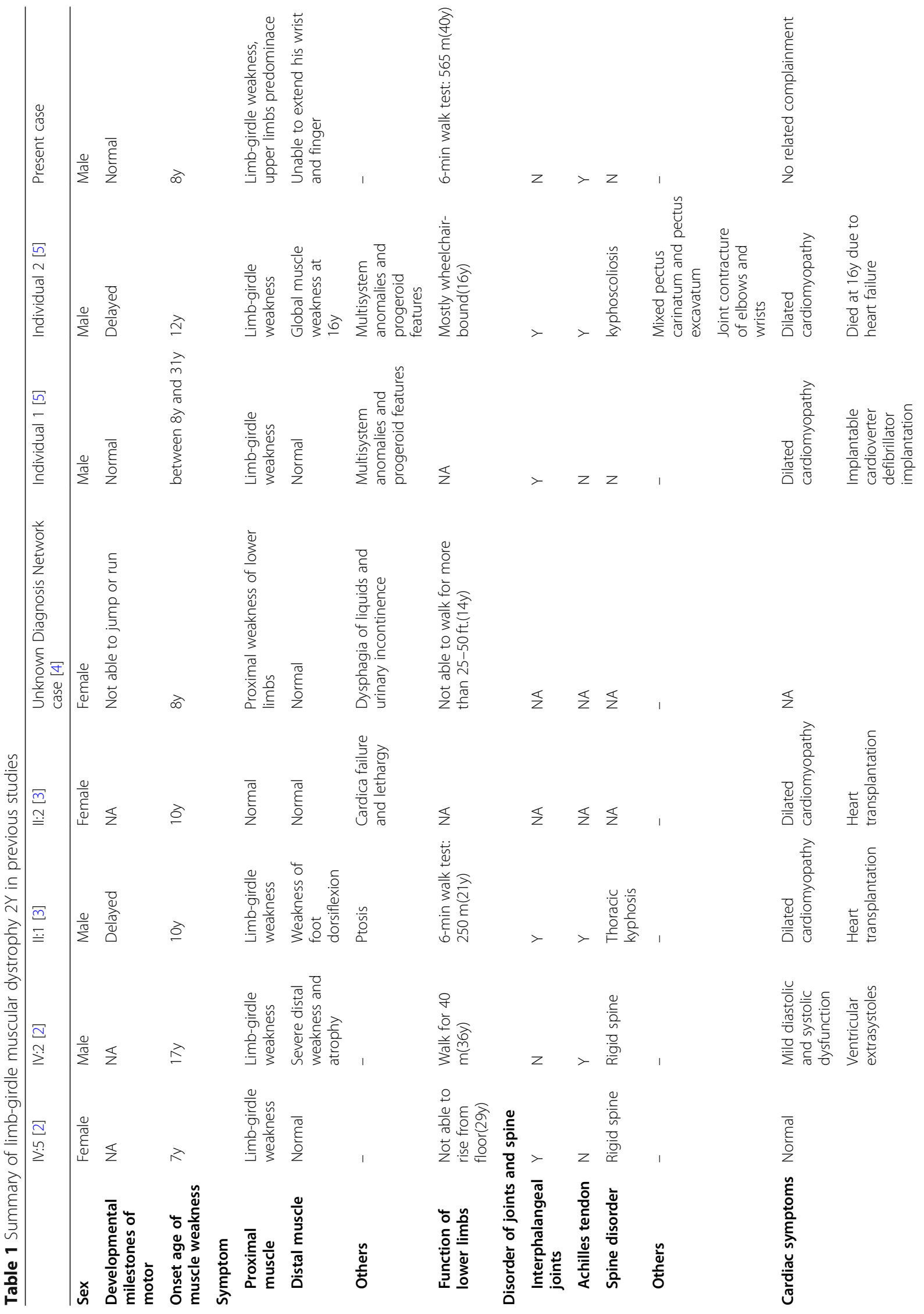




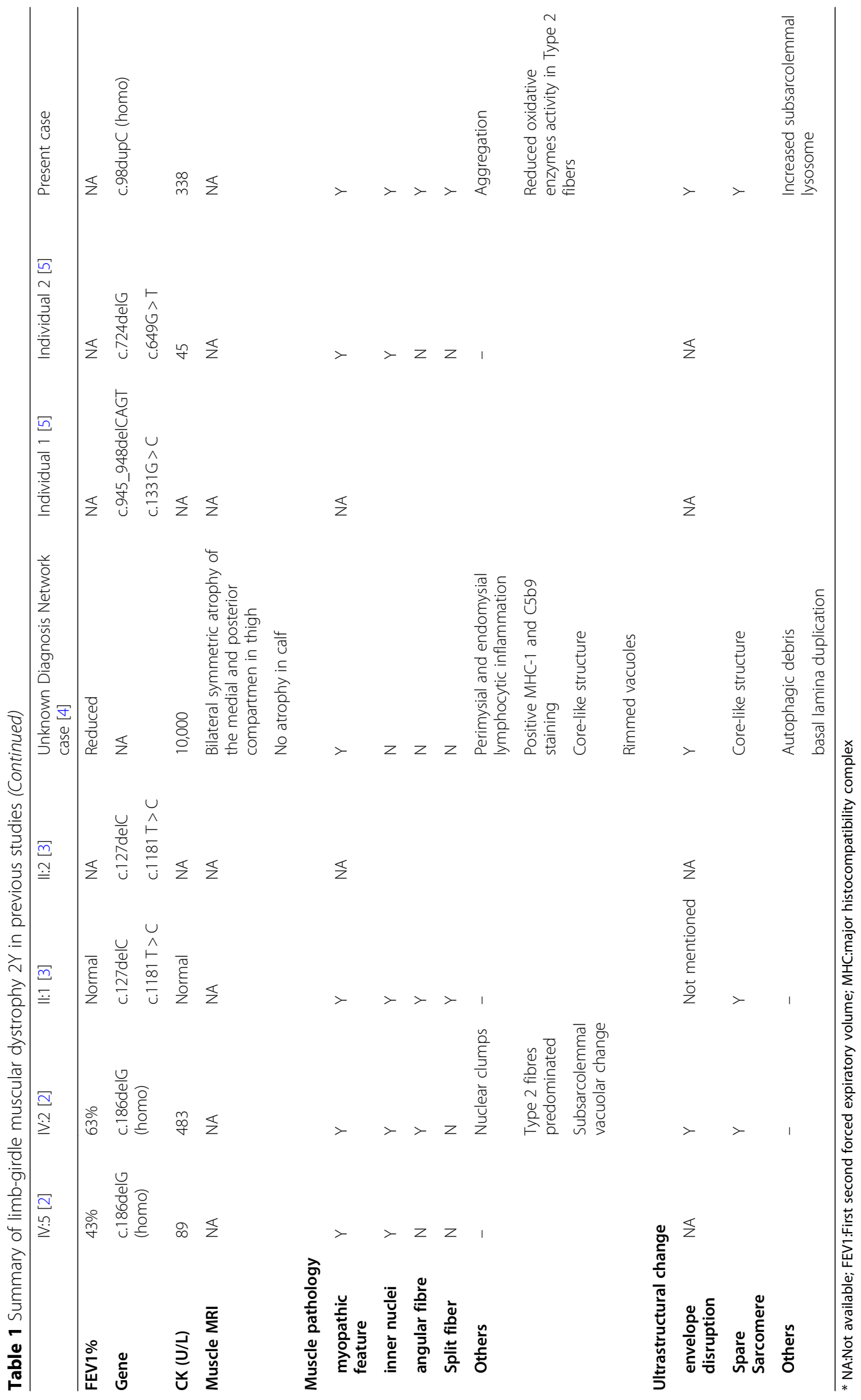


his lower limbs (6-min walk test: $565 \mathrm{~m}$, better than the previous case and no impairment of distal lower limbs), his upper limbs were severely affected (PUL score: 33 and unable to extend wrist and finger). In addition, axial muscle weakness was also noticed (decreased neck flexion strength).

In muscle pathology, most of the reported LGMD2Y cases had myopathic changes under a light microscope. Necrosis with inflammatory infiltration was uncommon but was also reported in a rapidly exacerbated case [4]. Ultrastructural alternation was mostly restricted in nuclei varying from chromatin condensation to envelope disruption and naked chromatin. Sarcomeric architecture was always spared. The specimens of the present patients also showed myopathic changes on light microscopy (Fig. 2a) and envelope disruption on electron microscopy (Fig. 3).

In addition to muscular dystrophy and cardiac involvement, mutation of TOR1AIP1 also resulted in dystonia [16], cerebellar atrophy [16], progeroid features and multisystem disorder [5, 17], which would also present with muscular dystrophy [5]. The heterogeneity of TOR1AIP1-related envelopathies may result from the two different isoforms of LAP1 in humans, LAP1B and LAP1C [18]. The absence of LAP1B was related to muscular dystrophy [2], and the loss of LAP1C was related to multisystem syndrome $[5,16,17]$.

Interestingly, the muscular dystrophy cases reported by Ghaoui et al. [3] was a heterozygote who had a LAP1Caffected allele and LAP1C-preserved allele. This may explain why their symptoms were restricted to skeletal muscle and cardiac muscle but also had more severe cardiomyopathy. In addition, the present case was less affected than other LAP1C-preserved patients considering his present lower limb function (GMW 1 vs. 5-8, compared with the case reported by Kayman-Kurekci et al. [2]). The mechanism of heterogeneity among muscular dystrophy cases is still not clear. The mutation of all reported muscular dystrophy cases was in the first exon, which encodes the intranuclear part of LAP1. Most of them were in the beginning of the peptide (position 3562), which may regulate the translation or post-translation modification of LAP1C. By regulating LAP1C, the difference in TOR1AIP1 mutation resulted in the heterogeneous presentation of muscular dystrophy cases.

LAP1 is a nuclear envelope-associated protein [6] that contributes to nuclear envelope maintenance. As a result of nuclear envelope alteration, LGMD2Y shares some common points with Emery-Dreifuss muscular dystrophy, which is also caused by mutations in nuclear envelope proteins, such as emerin [19] and nesprin [20]. Both of them featured limb girdle muscular dystrophy, joint contracture and cardiac involvement [21]. However, the mechanism of such a connection between this phenotype and nuclear envelope alteration is still not clear.
Interestingly, mutations in other nuclear envelope proteins, such as nesprin-1 [22], also resulted in central nervous system disorders similar to LAP1. In addition, the heterogeneity of nesprin-1 was also related to the mutation position and corresponding isoform [23]. The heterogeneity of nuclear envelope proteins and their connection with isoforms deserve further investigation.

Our study reported a case of LGMD2Y with relatively selective distal upper limb weakness and joint contracture. After our review of reported TOR1AIP1 mutation cases, we revealed the heterogeneity of LGMD2Y and the role of LAP1C.

\section{Abbreviations \\ GMW: Gardner-Medwin and Walton modified scale of clinical severity score; LAP1: lamina-associated polypeptide 1; LGMD: Limb-girdle muscular dystrophy; MRC: Medical Research Council; PUL: Performance of upper limbs; TOR1AIP1: torsinA-interacting protein 1}

\section{Acknowledgements}

Not applicable.

\section{Authors' contributions}

XLF analysed patient data and reviewed previous case. JLW analysed and interpreted electron microscope result. WBX finished muscle biopsy and patient evaluation. BL were responsible for interpretation muscle pathology. SJL finished electromyogram and analysed the result. XLY recruited patients and were responsible for diagnosis. FXL and WXJ were the major contributors in writing the manuscript. All authors had read and approved the final manuscript.

\section{Funding}

This study was supported by grants from the Southern China International Cooperation Base for Early Intervention and Functional Rehabilitation of Neurological Diseases (2015B050501003), Guangdong Provincial Engineering Center For Major Neurological Disease Treatment, Guangdong Provincial Translational Medicine Innovation Platform for Diagnosis and Treatment of Major Neurological Disease, Guangdong Provincial Clinical Research Center for Neurological Diseases and Natural Science Foundation of Guangdong Province (2017A030313850). The sponsor or funding organization had no role in the design or conduct of this research.

\section{Availability of data and materials}

The datasets used and/or analysed during the current study are available from the corresponding author on reasonable request.

\section{Ethics approval and consent to participate}

This study was performed in accordance with the Declaration of Helsinki and was approved under the guidelines of the Institutional Ethics Committee of the First Affiliated Hospital of Sun Yat-Sen University. Written informed consent for participation was obtained from the patient.

\section{Consent for publication}

Written informed consent for publication was obtained from the patient.

\section{Competing interests}

The authors declare that they have no competing interests.

\section{Author details}

'Department of Neurology, The First Affiliated Hospital, Sun Yat-sen University; Guangdong Provincial Key Laboratory of Diagnosis and Treatment of Major Neurological Diseases, National Key Clinical Department and Key Discipline of Neurology, No.58 Zhongshan Road 2, Guangzhou 510080, China. ${ }^{2}$ Laboratory of Electron Microscope, Zhongshan School of Medicine, Sun Yat-sen University, Guangzhou 510080, China. ${ }^{3}$ Department of Pathology, the First Affiliated Hospital, Sun Yat-sen University, Guangzhou 510080, China 
Received: 1 April 2020 Accepted: 27 August 2020

Published online: 01 September 2020

\section{References}

1. Guglieri M, Straub V, Bushby K, Lochmüller H. Limb-girdle muscular dystrophies. Curr Opin Neurol. 2008;21(5):576-84

2. Kayman-Kurekci G, Talim B, Korkusuz P, Sayar N, Sarioglu T, Oncel I, et al. Mutation in TOR1AIP1 encoding LAP1B in a form of muscular dystrophy: a novel gene related to nuclear envelopathies. Neuromuscul Disord [Internet] 2014;24(7):624-33 Available from: https://doi.org/10.1016/j.nmd.2014.04.007.

3. Ghaoui R, Benavides T, Lek M, Waddell LB, Kaur S, North KN, et al. TOR1AIP1 as a cause of cardiac failure and recessive limb-girdle muscular dystrophy. Neuromuscul Disord [Internet]. 2016;26(8):500-3 Available from: https://doi. org/10.1016/j.nmd.2016.05.013.

4. Bhatia A, Mobley BC, Cogan J, Koziura ME, Brokamp E, Phillips J, et al. Magnetic resonance imaging characteristics in case of TOR1AIP1 muscular dystrophy. Clin Imaging. 2019;58:108-13.

5. Lessel I, Jan M, Sabine C, Florian L, Sigrid A, Stefanie F, et al. Two nove cases further expand the phenotype of TOR1AIP1 - associated nuclear envelopathies. Hum genet [internet]. 2020;(0123456789). Available from. https://doi.org/10.1007/s00439-019-02105-6.

6. Kondo Y, Kondoh J, Hayashi D, Ban T, Takagi M, Kamei Y, et al. Molecular cloning of one isotype of human lamina-associated polypeptide $1 \mathrm{~s}$ and a topological analysis using its deletion mutants. Biochem Biophys Res Commun. 2002;294(4):770-8.

7. Stelzer G, Rosen N, Plaschkes I, Zimmerman S, Twik M, Fishilevich S, et al. The GeneCards suite: from gene data mining to disease genome sequence analyses. Curr Protoc Bioinforma [internet]. 2016;54. Available from. http:// www.ncbi.nlm.nih.gov/pubmed/27322403.

8. Walton JN. Gardner-Medwin D. Progressive muscular dystrophy and the myotonic disorders. In: JN W, editor. Disorders of voluntary muscles. 1981. p. p481-524.

9. ATS statement: guidelines for the six-minute walk test. Am J Respir Crit Care Med. 2002 Jul;166(1):111-7.

10. Brooke MH, Griggs RC, Mendell JR, Fenichel GM, Shumate JB, Pellegrino RJ. Clinical trial in Duchenne dystrophy. I. the design of the protocol. Muscle Nerve. 1981;4(3):186-97.

11. Mayhew A, Mazzone ES, Eagle M, Duong T, Ash M, Decostre V, et al. Development of the performance of the upper limb module for Duchenne muscular dystrophy. Dev Med Child Neurol. 2013;55(11):1038-45.

12. Li H. Durbin R. Fast and accurate short read alignment with BurrowsWheeler transform bioinformatics. 2009;25(14):1754-60

13. McKenna A, Hanna M, Banks E, Sivachenko A, Cibulskis K, Kernytsky A, et al. The genome analysis toolkit: a MapReduce framework for analyzing nextgeneration DNA sequencing data. Genome Res. 2010;20(9):1297-303.

14. Wang K, Li M, Hakonarson H. ANNOVAR: functional annotation of genetic variants from high-throughput sequencing data. Nucleic Acids Res. 2010; 38(16):e164.

15. Richards S, Aziz N, Bale S, Bick D, Das S, Gastier-Foster J, et al. Standards and guidelines for the interpretation of sequence variants: a joint consensus recommendation of the American College of Medical Genetics and Genomics and the Association for Molecular Pathology. Genet Med. 2015; 17(5):405

16. Dorboz I, Coutelier M, Bertrand AT, Caberg JH, Elmaleh-Bergès M, Lainé J, et al. Severe dystonia, cerebellar atrophy, and cardiomyopathy likely caused by a missense mutation in TOR1AIP1. Orphanet J Rare Dis. 2014:9:174.

17. Fichtman B, Zagairy F, Biran N, Barsheshet Y, Chervinsky E, Ben Neriah Z, et al. Combined loss of LAP1B and LAP1C results in an early onset multisystemic nuclear envelopathy. Nat Commun [Internet]. 2019;10(1). Available from: https://doi.org/10.1038/s41467-019-08493-7.

18. Santos M, Domingues SC, Costa P, Muller T, Galozzi S, Marcus K, et al. Identification of a novel human LAP1 isoform that is regulated by protein phosphorylation. PLoS One. 2014;9(12):e113732.

19. Nagano A, Koga R, Ogawa M, Kurano Y, Kawada J, Okada R, et al. Emerin deficiency at the nuclear membrane in patients with Emery-Dreif uss muscular dystrophy. Nat Genet. 1996;12(3):254-9

20. Zhang Q, Bethmann C, Worth NF, Davies JD, Wasner C, Feuer A, et al. Nesprin-1 and-2 are involved in the pathogenesis of Emery-Dreifuss muscular dystrophy and are critical for nuclear envelope integrity. Hum Mo Genet. 2007;16(23):2816-33.
21. Worman HJ, Dauer WT. The nuclear envelope: an intriguing focal point for Neurogenetic disease. Neurotherapeutics. 2014;11(4):764-72.

22. Noreau A, Bourassa CV, Szuto A, Levert A, Dobrzeniecka S, Gauthier J, et al. SYNE1 mutations in autosomal recessive cerebellar ataxia. JAMA Neurol. 2013;70(10):1296-301.

23. Peng $Y$, Ye W, Chen Z, Peng H, Wang P, Hou X, et al. Identifying SYNE1 Ataxia with novel mutations in a Chinese population. Front Neurol. 2018; 9(December): $1-10$.

\section{Publisher's Note}

Springer Nature remains neutral with regard to jurisdictional claims in published maps and institutional affiliations.
Ready to submit your research? Choose BMC and benefit from:

- fast, convenient online submission

- thorough peer review by experienced researchers in your field

- rapid publication on acceptance

- support for research data, including large and complex data types

- gold Open Access which fosters wider collaboration and increased citations

- maximum visibility for your research: over $100 \mathrm{M}$ website views per year

At $\mathrm{BMC}$, research is always in progress.

Learn more biomedcentral.com/submissions 